\title{
A Rare Presentation of Low-grade Appendiceal Mucinous Neoplasm Within an Amyand's Hernia: A Case Report
}

\author{
Hani Atiqah Saim, Chik Ian, Fahrol Fahmy Jaafar, Zamri Zuhdi, Razman Jarmin, Azlanudin Azman \\ Department of Surgery, Universiti Kebangsaan Malaysia Medical Centre, Kuala Lumpur, Malaysia
}

\begin{abstract}
An Amyand's hernia is characterised as the presence of the appendix in an inguinal hernial sac. During laparoscopic cholecystectomy for gallbladder polyps, an incidental Amyand's hernia was discovered in a 75-year-old female patient. On examination, the hernia contained an appendiceal mucocele but no evidence of perforation. An open appendicectomy with tension-free mesh repair was performed for the hernia. The histopathological report of the appendix was a low-grade appendiceal mucinous neoplasm (LAMN), an entity that is just as rare as an Amyand's hernia. The patient had the right inguinal swelling for over 10 years but it was thought to be an inguinal intramuscular cyst as reported on a previous abdominal ultrasound. Mucocele of the appendix may have a benign or malignant appendiceal progress, leading to individualised diagnosis and treatment. We review an Amyand's hernia with LAMN and discuss the asymptomatic tendency yet malignant potential of appendiceal mucoceles along with treatment strategies.
\end{abstract}

Keywords: Neoplasms; Mucocele; Appendix; Appendicitis; Hernia

\section{INTRODUCTION}

Amyand's hernia was first described by a French surgeon, Claudius Amyand, in an 11-year-old boy who underwent a successful appendectomy in 1735 [1]. The incidence of finding an appendix within the hernia sac is rare, occurring in less than $1 \%$ of inguinal hernia patients. When complications arise such as inflammation, perforation, or abscess formation it becomes exceedingly rare with an incidence of around $0.1 \%$ [2]. Appendiceal mucocele is a cystic mass that develops due to mucus accumulation in the lumen of the appendix. Although they occur at a low rate of $0.2 \%$ to $0.7 \%$, rupture of an appendiceal mucocele, may it be spontaneous or iatrogenic, can cause mucinous intraperitoneal ascites, a clinical condition known as pseudomyxoma peritonei

Received: Jun 3,2021 - Revised: Jul 5, 2021 - Accepted: Jul 13, 2021 Correspondence to: Azlanudin Azman, MBBchBAO

Department of Surgery, Level 8, Universiti Kebangsaan Malaysia Medical Centre, Jalan Yaacob Latif, Bandar Tun Razak, 56000 Kuala Lumpur,

Malaysia

Tel: +60-192817878, Fax: +60-391456684

E-mail:drazlan@ppukm.ukm.edu.my

ORCID: https://orcid.org/0000-0002-5897-3134

(C) 2021 The Korean Society of Coloproctology

This is an open-access article distributed under the terms of the Creative Commons Attribution NonCommercial License (https://creativecommons.org/licenses/by-nc/4.0) which permits unrestricted noncommercial use, distribution, and reproduction in any medium, provided the original work is properly cited.
(PMP), which is associated with high mortality [3]. Other complications of appendicular mucocele include intestinal bleeding, intestinal obstruction, and pyonephrosis. Appendicular mucocele has a higher occurrence in females and people over the age of 50 years [4].

We present a case of 2 rare entities; an Amyand's hernia and lowgrade appendiceal mucinous neoplasm (LAMN). Amyand's hernia was diagnosed intraoperatively during laparoscopic cholecystectomy. On closer examination, the hernia contained an appendiceal mucocele with no evidence of perforation. After completion of the laparoscopic cholecystectomy, the patient subsequently underwent an appendicectomy and tension-free repair with mesh. The appendiceal histopathology examination revealed a LAMN. In this article, we review the relevant literature.

This case report has been reported in line with the SCARE (Surgical CAse Report) criteria [4]. Written informed consent was obtained from the patient for publication of this case report and accompanying images.

\section{CASE REPORT}

Our patient was a fit 75-year-old female who initially presented with epigastric pain and vomiting. She was diagnosed with biliary colic and scheduled for an elective laparoscopic cholecystectomy. Incidentally, on one of her presentations to the emergency depart- 


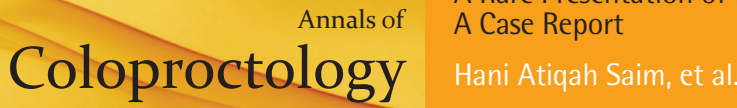

ment for biliary colic, she complained of occasional pain over a progressively increasing right inguinal region swelling. According to her, it has been present for the past 10 years with no history of reducibility. An abdominal ultrasound was performed which showed a right inguinal intramuscular cystic lesion measuring $3.2 \times 4.3 \times 3.5 \mathrm{~cm}$ with layering within that may represent a hematoma or collection (Fig. 1). As she was already scheduled for surgery, it was decided for her to undergo an open excisional biopsy of the right inguinal cyst in the same setting as the laparoscopic cholecystectomy.

However, during the initial laparoscopic examination for the laparoscopic cholecystectomy, we noted that there was omentum adhered to the anterior abdominal wall at the right iliac fossa region which raised the suspicion of an irreducible inguinal hernia containing omentum. We concluded that the so-called inguinal cystic lesion seen on ultrasound was actually a misdiagnosed irreducible inguinal hernia and decided to perform an open hernia repair after completion of the laparoscopic cholecystectomy. During repair of the inguinal hernia, we found that it contained the appendix within its sac (Fig. 2). The appendix measured 7.0 $\times$ $3.5 \times 2.5 \mathrm{~cm}$ with the presence of cystic dilation of the tip measuring $5.0 \times 3.5 \times 2.5 \mathrm{~cm}$. On the cut section, the dilated part was filled with gelatinous mucinous material. The surrounding wall was healthy and no perforation was seen. There was no mucin seen on the outer wall either. These findings conclude that it was an appendiceal mucocele.

The patient was discharged on the third postoperative day after an uneventful recovery and remained asymptomatic. Histopathology examination revealed a LAMN, pathologic Tis (pTis). The histopathology of the gallbladder was unremarkable revealing only chronic cholecystitis with cholesterol polyps. Sections

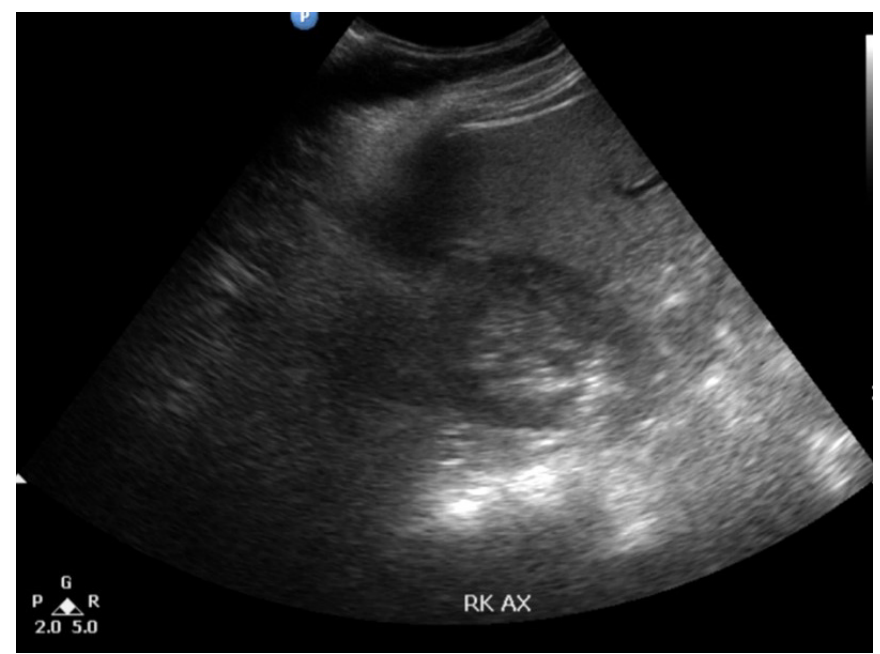

Fig. 1. Ultrasonography of the abdomen revealing a right inguinal cystic lesion containing echogenic layering within. from the dilated portion of the appendix showed a LAMN confined to the muscularis mucosa (Fig. 3). The tumor showed flat, single layer mucinous epithelium associated with extensive mucosal ulceration replaced by acellular mucin, dense lymphoplasmacytic infiltration, and foreign body-type multinucleated giant cells (Fig. 4). It was composed of columnar epithelium with apical mucin and elongated, hyperchromatic, pseudostratified nuclei with inconspicuous nucleoli. No mitosis was seen. In areas, the muscularis mucosa was attenuated and replaced by fibrotic and hyalinized stroma.

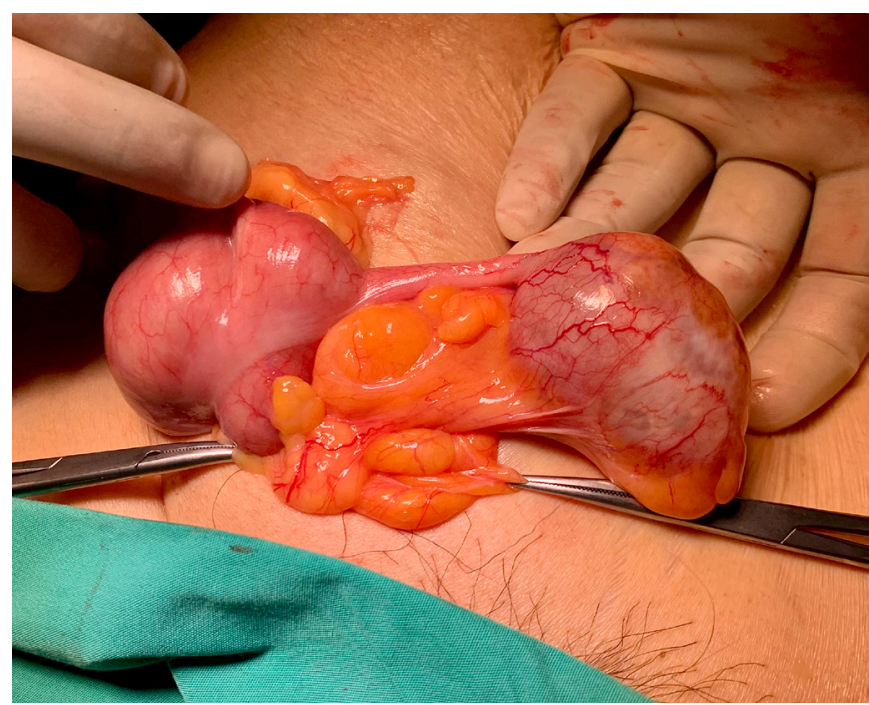

Fig. 2. Intraoperative image of the appendiceal mucocele within the hernia.

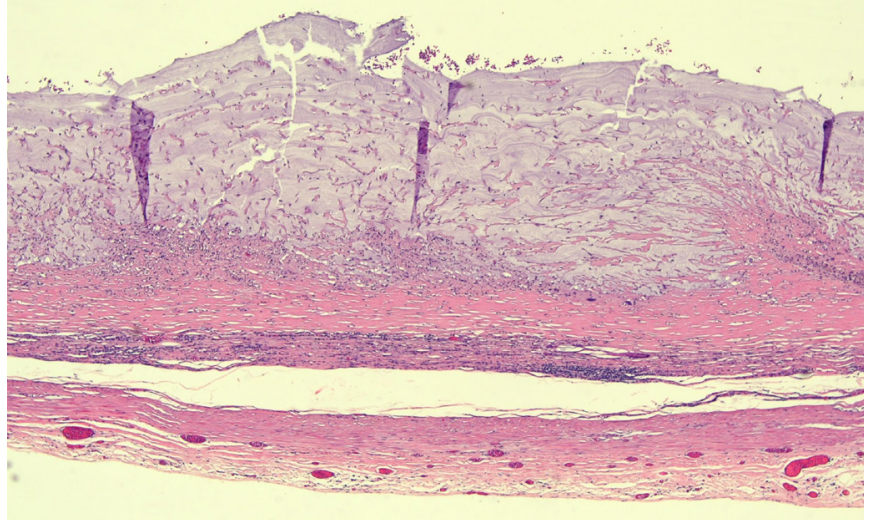

Fig. 3. The appendix is lined by columnar epithelium with apical mucin and elongated, hyperchromatic, pseudostratified nuclei with inconspicuous nucleoli, associated with attenuation of the muscularis propria $(\mathrm{H} \& \mathrm{E}, \times 10)$. 


\section{DISCUSSION}

Protrusion of a vermiform appendix in an inguinal hernia sac is known as an Amyand's hernia. Since the first description by Claudius Amyand, there have been many iterations of classifying this entity in order to guide its management. Losanoff and Basson [5] divided their classification of Amyand's hernias into 4 subtypes: (1) normal appendix within the inguinal hernia, (2) hernia with inflamed appendicitis, (3) hernia with perforation of appendicitis, and (4) complications including abscess or malignancy. This has allowed surgeons to decide on the best mode of treatment for their patients depending on the presentation. Singal and Gupta [6] further modified this classification to include Amyand hernias in an incisional hernial sac, as demonstrated in Table 1. Nevertheless, the principles behind the management strategies remain the same.

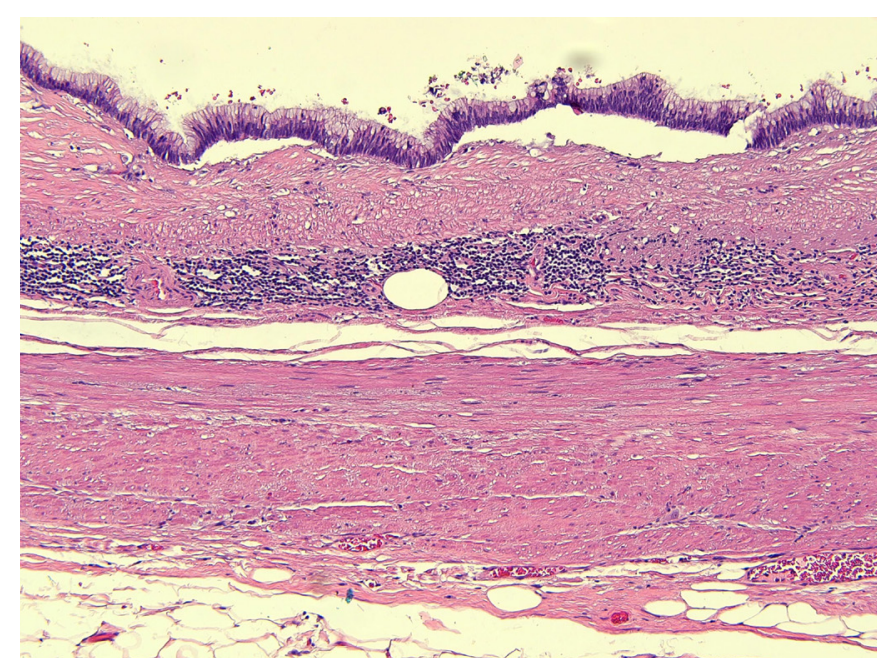

Fig. 4. Dissecting mucin. Extensive mucosal ulceration replaced by acellular mucin, dense lymphoplasmacytic infiltration, and foreign body-type multinucleated giant cells $(\mathrm{H} \& \mathrm{E}, \times 10)$.
In cases of Amyand's hernia with appendicitis or perforation, the management should be appendicectomy. However, in cases where the appendix is normal and without any inflammation, most believe appendicectomy may not be necessary. The appendix may be reduced, and hernia repair is done with a mesh. Some recommend appendicectomy in all cases of Amyand's hernia because they believe that the appendix is prone to shift positions within the hernia, and manipulation of the appendix during reduction may lead to appendicitis [7]. In all cases of uninflamed Amyand's hernia, hernia repair with mesh is acceptable.

Preoperative diagnosis of Amyand's hernia, although difficult clinically, is feasible with ultrasound and computed tomography (CT). On ultrasound imaging, a blind-ended tubular structure with thickened walls in connection with the caecum inside the hernia sac is suggestive of an Amyand's hernia. CT allows direct visualization of the appendix inside the inguinal canal. Even if this is not possible, the location of the caecum in proximity to the hernia sac is indicative of Amyand's hernia [2]. Barium enemas have also been reported to diagnose this condition. If a diagnosis is acquired preoperatively, information from these imaging modalities can be useful in operative design.

Appendicular mucocele has an incidence of $0.2 \%$ to $0.3 \%$ with a higher prevalence in females and people over the age of 50 years. It is an obstructive dilatation of the appendix caused by intraluminal accumulation of mucoid material. Preoperative diagnosis of appendicular mucocele is not easy because of the rarity of the condition and the non-specific nature of the presenting symptoms. Fifty percent of cases present with a palpable mass [2]. Abdominal ultrasound and CT scans are important diagnostic tools, but histopathology is needed for a definitive diagnosis. Open surgery is preferred to the laparoscopic approach as the latter increases the risk of rupture. However, laparoscopic appendicectomy is still performed on certain patients. An algorithm for the selection of the type of surgery has been coined by Dhage-Ivatury and Sugarbaker [8]. Simple appendectomy is the choice for patients with benign mucocele as suggested by the presence of a

Table 1. Singal and Gupta's classification of Amyand's hernia [5]

\begin{tabular}{|c|c|c|}
\hline Classification & Description & Surgical management \\
\hline Type 1 & Normal appendix within an inguinal hernia & Hernia reduction, mesh repair, appendicectomy in young patients \\
\hline Type 2 & Acute appendicitis within an inguinal hernia, no abdominal sepsis & Appendicectomy through hernia, primary repair of hernia, no mesh \\
\hline Type 3 & $\begin{array}{l}\text { Acute appendicitis within an inguinal hernia, abdominal wall, or peritoneal } \\
\text { sepsis }\end{array}$ & Laparotomy, appendicectomy, primary repair of hernia, no mesh \\
\hline Type 4 & $\begin{array}{l}\text { Acute appendicitis within an inguinal hernia, related or unrelated } \\
\text { abdominal pathology }\end{array}$ & $\begin{array}{l}\text { Manage as types } 1 \text { to } 3 \text { hernia, investigate or treat second pathology as } \\
\text { appropriate }\end{array}$ \\
\hline Type 5a & Normal appendix within an incisional hernia & Appendicectomy through hernia, primary repair of hernia including mesh \\
\hline Type 5b & Acute appendicitis within an incisional hernia, no abdominal sepsis & Appendicectomy through hernia, primary repair of hernia \\
\hline Type 5c & $\begin{array}{l}\text { Acute appendicitis within an incisional hernia, abdominal wall, or } \\
\text { peritoneal sepsis or in relation to previous surgery }\end{array}$ & Manage as type 4 \\
\hline
\end{tabular}

Adapted from Singal and Gupta [6] with permission of MÆEDICA - a Journal of Clinical Medicine. 
normal caecum and appendicular base and no evidence of perforation, as in our patient. Right hemicolectomy is recommended when a malignant mucocele is suspected by the presence of a perforated mucocele, enlarged mesenteric lymph node, or positive cytology. Surgery for benign appendicular mucoceles has an excellent long-term prognosis [7].

LAMNs have the potential to progress to PMP via the peritoneal spread. The presence of mucin or neoplastic epithelium outside the lumen, in places such as the appendiceal submucosa, wall, or periappendiceal tissue, is associated with PMP. Lesions of this type can be labeled as LAMN type 2. Lesions with disease confined to the lumen are defined as LAMN type 1 [9]. The 8th edition American Joint Committee on Cancer staging manual provides guidelines for staging both LAMNs and adenocarcinomas. LAMNs that are confined to the appendiceal wall without invasion or loss of the muscularis propria are classified as Tis LAMN. This is to reflect the excellent outcome for confined LAMNs.

In our case, Amyand's hernia was not diagnosed preoperatively because her history of the inguinal lump was not suggestive of a hernia and further imaging such as a CT scan was not performed as it was not indicated. The presence of a mucocele at the tip of the appendix within the hernia sac was probably the reason an impression of an intramuscular cystic lesion was given in the preoperative ultrasound. Our decision to perform an open appendicectomy and a mesh repair of the hernia was deemed correct as the appendix within the inguinal hernial sac turned out to be an appendiceal mucocele and histologically proven LAMN, pTis. Laparoscopic manipulation of the appendix would have increased the risk of appendicular rupture and the feared complication of PMP due to gradual intraperitoneal accumulation of mucin.

Oncologic outcomes after appendectomy, including the entire mesoappendix for LAMN without perforation or peritoneal involvement, have shown very low recurrence rates, as these neoplasms have a slow-moving behavior [10]. Appropriate initial surgical management is of utmost importance because iatrogenic rupture of the appendix can transform the situation from localized to disseminate. On that account, if an unruptured LAMN cannot be safely resected laparoscopically, conversion to an open operation is recommended. Limited published data suggest that a microscopically positive resection margin after appendectomy for non-perforated LAMN does not predict disease recurrence and therefore does not warrant formal right hemicolectomy [11]. Although there are no formal surveillance guidelines for appendiceal neoplasms after appendectomy, patients with low-grade localized tumors of the appendix who undergo appendectomy alone rarely develop PMP; therefore, frequent postoperative imaging for extended intervals is typically of minimal benefit [12].

In conclusion, identification of Amyand's hernia is crucial and neoplasm of the appendix should always be considered in patients with a right inguinal mass. It is the responsibility of the surgeon, aided by radiologists and emergency physicians. Preoperative diagnosis of this rare entity is not straightforward and is often an in- cidental finding during surgery. If the diagnosis is acquired preoperatively, it will aid the surgeon in planning an optimal operative design. Nevertheless, surgical treatment by an open appendicectomy and hernia repair with a synthetic mesh is appropriate if diagnosed intraoperatively.

\section{CONFLICT OF INTEREST}

No potential conflict of interest relevant to this article was reported.

\section{ACKNOWLEDGMENTS}

The authors acknowledge Dr. Wan Syahira Ellani Wan Ahmad Kammal and Dr. Nor Hafizah Mohd Zain from Department of Pathology, Universiti Kebangsaan Malaysia Medical Centre.

\section{REFERENCES}

1. Hutchinson R. Amyand's hernia. J R Soc Med 1993;86:104-5.

2. Michalinos A, Moris D, Vernadakis S. Amyand's hernia: a review. Am J Surg 2014;207:989-95.

3. Rymer B, Forsythe RO, Husada G. Mucocoele and mucinous tumours of the appendix: a review of the literature. Int J Surg 2015; 18:132-5.

4. Agha RA, Fowler AJ, Saeta A, Barai I, Rajmohan S, Orgill DP, et al. The SCARE statement: consensus-based surgical case report guidelines. Int J Surg 2016;34:180-6.

5. Losanoff JE, Basson MD. Amyand hernia: a classification to improve management. Hernia 2008;12:325-6.

6. Singal R, Gupta S. "Amyand's hernia" - pathophysiology, role of investigations and treatment. Maedica (Bucur) 2011;6:321-7.

7. Saad EA, Elsamani EY, AbdElrahim WE, Elsiddig KE, Khalil EA. Surgical treatment of mucocele of the appendix: a systematic review and case report. J Surg Case Rep 2018;2018:rjy102.

8. Dhage-Ivatury S, Sugarbaker PH. Update on the surgical approach to mucocele of the appendix. J Am Coll Surg 2006;202:680-4.

9. Demetrashvili Z, Chkhaidze M, Khutsishvili K, Topchishvili G, Javakhishvili T, Pipia I, et al. Mucocele of the appendix: case report and review of literature. Int Surg 2012;97:266-9.

10. Fournier K, Rafeeq S, Taggart M, Kanaby P, Ning J, Chen HC, et al. Low-grade appendiceal mucinous neoplasm of uncertain malignant potential (LAMN-UMP): prognostic factors and implications for treatment and follow-up. Ann Surg Oncol 2017;24:187-93.

11. Arnason T, Kamionek M, Yang M, Yantiss RK, Misdraji J. Significance of proximal margin involvement in low-grade appendiceal mucinous neoplasms. Arch Pathol Lab Med 2015;139:518-21.

12. Tiselius C, Kindler C, Shetye J, Letocha H, Smedh K. Computed tomography follow-up assessment of patients with low-grade appendiceal mucinous neoplasms: evaluation of risk for pseudomyxoma peritonei. Ann Surg Oncol 2017;24:1778-82. 